\title{
Coherent addition of two dimensional array of fiber lasers
}

\author{
Moti Fridman Vardit Eckhouse Nir Davidson Asher A. Friesem \\ Dept. of Physics of Complex system, Weizmann institute of Science, Rehovot 76100, \\ Israel
}

Elena Luria Vladimir Krupkin

ELOP, ElectroOptics Industries LTD. P.O. Box 1165, Rehovot 76111, Israel

\begin{abstract}
Configurations for efficient free space coherent addition of four separate fiber lasers arranged in two dimensional array are presented. They include compact and robust interferometric combiners that can be inserted either inside or outside the cavity of the combined lasers system. The results reveal that over $85 \%$ combining efficiency can be obtained.
\end{abstract}

Key words: fiber lasers, fiber array, coherent addition

PACS: $140.3510,140.3290,030.1640$

\section{Introduction}

Several approaches have been developed for phase locking and coherent addition of fiber lasers in order to generate a combined laser with high output power and high brightness. Some of these involve active phase locking [1], Talbot self imaging [2,3], evanescent wave coupling [4], and spatial filters [5,6,7], for obtaining phase locking within the laser cavity and subsequent external 
coherent addition of all phased locked lasers. In these the brightness of the combined far field spot is relatively low because of the inherent separations between the lasers. Other approaches involve direct coherent addition within the lasers cavities by inserting intra-cavity fiber couplers [8,9]. Although in these direct approaches the alignment is relatively easy and the stability is good, the combined output power is rather limited in order to prevent damage and undesirable non-linear effects to a single output fiber.

To overcome damage and deleterious non-linearities to the output fibers, it is possible to resort to configurations in which the coherent addition is performed in free space with robust intracavity interferometric combiners. Such configurations were already effectively demonstrated for coherent addition of several solid state lasers whose cavity lengths and alignments could be readily controlled [10,11]. With fiber lasers, whose cavity lengths could not be controlled with sufficient accuracy and alignment is difficult, the situation is much more complicated. Earlier attempts with configurations in which beam splitters in free space were exploited, successfully demonstrated coherent addition of two fiber lasers [12,13]. Unfortunately, such configurations cannot be easily upscaled because they are bulky and difficult to align.

In this article, we present two robust, stable, highly efficient, free-space configurations for coherently adding four fiber lasers arranged in two dimensional array. In these configurations coherent addition is performed by means of compact interferometric combining elements in free space. Such configurations could be readily upscaled to coherently add many fiber lasers.

\section{Experimental Configuration and Procedure}

We considered two different configurations for free-space coherent addition of four fiber lasers. The first, namely the intra-cavity 
configuration, is presented schematically in Fig. 1. It included four fiber lasers operating at $1550 \mathrm{~nm}$ and two intra-cavity interferometric combiners. Each fiber laser consisted of double-clad single-mode Erbium doped fiber of about 6 meters in length, where one end was attached to a high reflection fiber Bragg grating (FBG) of $5 \mathrm{~nm}$ spectral bandwidth around $1550 \mathrm{~nm}$ that served as a back reflector mirror and the other end was spliced to a collimating graded index (GRIN) lens with anti-reflection layer to suppress any reflections back into the fiber cores, and a flat output coupler of $20 \%$ reflection that was common to all fiber lasers. A focusing lens was inserted in front of the output coupler for controlling the coupling strength between the lasers. Each fiber laser was pumped with a multi-mode diode laser of $915 \mathrm{~nm}$ wavelength, that was spliced to the back of the FBG. The interferometric combiner was a planar substrate, where half of the front surface was coated with an antireflection layer and the other half with a $50 \%$ beam-splitter layer, while half of the rear surface was coated with a highly reflecting layer and the other half with an antireflection layer [11].

We measured the spectrum of each fiber laser separately with a fast photo-detector connected to an RF spectrum analyzer. From these spectra we determined that the overall lengths of the four laser cavities were $6.33 \mathrm{~m}, 6.41 \mathrm{~m}, 6.52 \mathrm{~m}$ and $6.64 \mathrm{~m}$, and that the relative intensity noise (RIN) was less than $-70 d B / H z$. We also measured the output power of each fiber laser as a function of the diode pump power, and found that the light-to-light efficiency was about $10 \%$ for each.

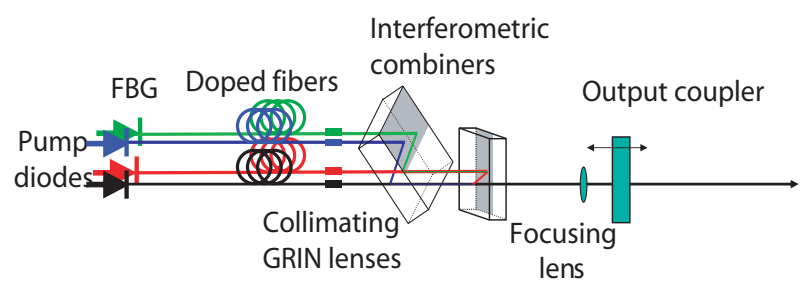

Fig. 1. (color online) Basic intra-cavity configuration for coherent addition of four fiber lasers in free space using two orthogonally oriented interferometric combiners.

The second configuration, namely the outer-cavity configuration, 
is presented schematically in Fig. 2. It differs from that shown in Fig. 1 in that an intermediate coupler of $20 \%$ was inserted at the output of the fiber lasers, so as to obtain efficient lasing from each, and the interferometric combiners were outside the cavities of the four individual lasers. Coupling between the lasers and consequent phase locking and coherent addition was achieved by the feedback to each fiber laser from the common output coupler. In both configurations, when the fiber lasers are phase locked, the first interferometric combiner transforms efficiently four light beams to two beams and the second interferometric combiner transforms the two beams into one nearly gaussian beam.

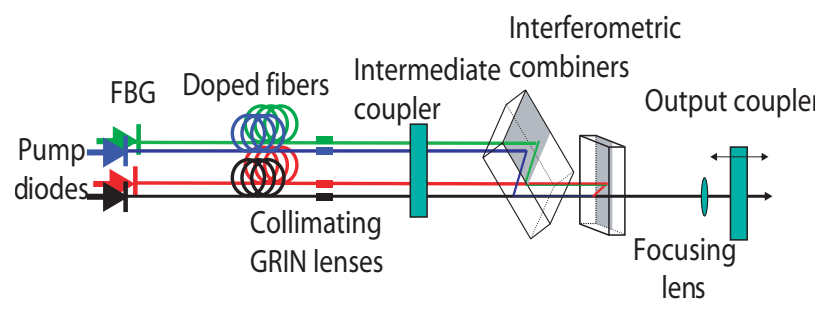

Fig. 2. (color online) Basic outer-cavity configuration for coherent addition of four fiber lasers in free space using two orthogonally oriented interferometric combiners.

To ensure that the beams emerging from the four individual fibers are exactly parallel, we first aligned and set one fiber in a mounting arrangement with respect to a planar output coupler so as to obtain optimal lasing. Next, we inserted the second fiber laser and aligned it with micrometer stages so it will also optimally lase from the same output coupler, and bonded it to the same mounting arrangement with UV cured optical cement. This procedure was repeated for the third and then fourth fiber lasers. We then measured the beam parallelism and ascertained that it is better than $0.1 \mathrm{mrad}$. The output light power from the individual fiber lasers were adjusted to be about the same by controlling their individual pump powers. 


\section{Experimental Results}

We began our experiments by performing spectral characterization of the combined output beam using a fast photo-detector connected to a spectrum analyzer, in order to determine the location and distribution of the common longitudinal modes [12]. A representative spectrum of the outer-cavity configuration, that contains four cavities of different lengths, each comprised of three mirrors, is presented in Fig. 3. As evident, there are many common bands of longitudinal modes that are needed for phase locking and coherent addition in both configurations, where the separation between the bands is $700 M H Z$ which corresponds to $20 \mathrm{~cm}$ length difference. This is the distance between the intermediate coupler and the output coupler. We also measured the spectrum for the simpler intra-cavity configuration and verified that there are even more common bands of longitudinal modes. When upscaling to a large number of fiber lasers, it is still an open question whether it would be possible to find common longitudinal modes.

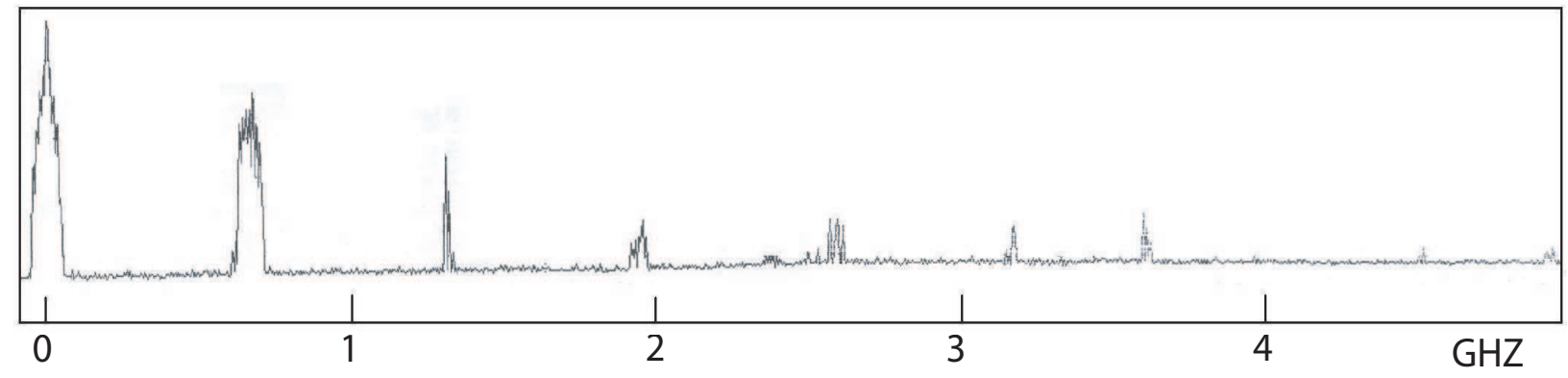

Fig. 3. Representative spectrum of the output beam from the outer-cavity configuration. Clear structure of lobs can be seen, indicating common longitudinal modes needed for phase locking and coherent addition.

Next we determined the intermediate and final combining efficiencies in the two configurations, i.e the ratio of the output power of the combined laser beam over the sum of the output power of the individual laser beams. This was done by first inserting only one interferometric combiner in order to coherently add horizontally the light from the four fiber lasers, each with a power of $35 \mathrm{~mW}$, to form two beams, each with a power of about $66 \mathrm{~mW}$. This corresponds to a combining efficiency of over $90 \%$. Then, we added 
the second interferometric combiner to coherently add vertically the two remaining beams into one beam to obtain a combined output power of about $121 \mathrm{~mW}$. This corresponds to an overall combining efficiency (i.e. the ratio between the overall power with the interferometric combiners to that without the combiners) of $86 \%$. The results were essentially the same for both configurations with a somewhat lower combining efficiency for the outer-cavity configuration, but still of over $80 \%$.

We also placed MicronViewer 7290 infrared cameras to detect the intensity distributions before the interferometric combiners, after the first interferometric combiner and after the second one. Representative results are shown in Fig. 4. As evident, the initial four beams first coherently transform to two beams after the first interferometric combiner and then to one beam after the second interferometric combiner.

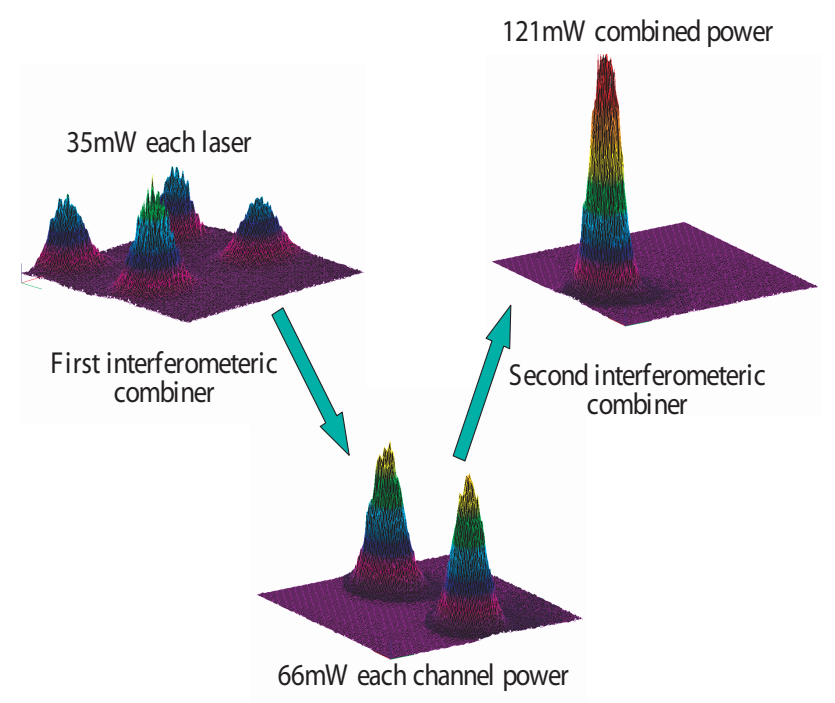

Fig. 4. (color online) Experimental intensity distributions for the intra-cavity configuration. (a) Before the interferometric combiners; (b) after the first interferometric combiner; (c) after the second interferometric combiner.

Finally, we measured the combined output power and determined the combining efficiency as a function of the coupling strength, namely the relative amount of light that is re-injected from one laser into the other. The coupling strength was accurately controlled by displacing the common output coupler in and out of 
the focal plane of a focusing lens which was inserted in front of the output coupler [13]. Maximum coupling strength of $5 \%$ was achieved when the output coupler was placed at the focal plane of the lens. The results are presented in Fig. 5. Figure 5 (a) shows the combining efficiency, which is also a measure of the phase locking between the lasers, as a function of the coupling strength. As evident, for the intra-cavity configuration the lasers are strongly phase locked (high combining efficiency) even at a very low coupling strength of about $0.5 \%$, whereas for the outer-cavity configuration strong phase locking occurs at a higher coupling strength of $2 \%$. Figure 5 (b) shows the combined output power as a function of coupling strength. As evident, the output power is essentially the same over a large range of coupling strengths.

At the lower coupling strengths, the decrease in output power for the outer-cavity configuration is due to reduction in phase locking, whereby more light is lost at the interferometric combiners. For the intra-cavity configuration, the reduction of output power is due to less efficient lasing because less light is reflected from the single common output coupler. These results extend our earlier results for only two fiber lasers [12,13] and indicate that it is possible to upscale phase locking and coherent addition to a larger number of fiber lasers.
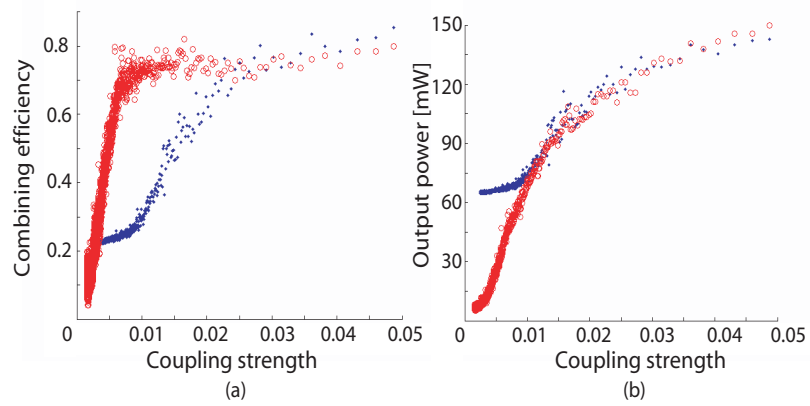

Fig. 5. (color online) Experimental combining efficiency and output power as a function of the coupling strength for the intra-cavity and outer-cavity configurations. Circles denote the results for the intra-cavity configuration. Dots denote the results for the outer-cavity configuration 


\section{Concluding Remarks}

To conclude, we presented two compact and robust configurations for efficient coherent addition in free space of four separate fiber lasers that are arranged in a two-dimensional array, using two binary interferometric combiners. We obtained high combining efficiency together with a good beam quality for both configurations. Although scalability to coherent addition of very large arrays of lasers is still uncertain [14], we believe that our approach could be extended to moderate arrays size. This could be done by either resorting to more binary interferometric combiners or two more complex interferometric combiners where each can coherently add more than two fiber lasers.

This research was supported in part by the Binational Science Foundation.

\section{References}

[1] S. J. Augst, T. Y. Fan, and A. Sanchez. Opt. Lett., 29, 474, 2004.

[2] M. Wrage, P. Glas, D. Fischer, M. Leitner, N. N. Elkin, D. V. Vysotsky, A. P. Napartovich, and V. N. Troshchiev. Opt. Comm., 205, 367, 2002.

[3] X. Zhu, A. Schlzgen, L. Li, H. Li, V. L. Temyanko, J. V. Moloney, and N. Peyghambarian. Opt. Exp., 15, 10340, 2007.

[4] L. Michaille, C. R. Bennett, D. M. Taylor, T. J. Shepherd, J. Broeng, H. R. Simonsen, and A. Petersson. Opt. Lett., 30, 1668, 2005.

[5] L. Liu, Y. Zhou, F. Kong, and Y. C. Chen. Appl. Phys. Lett., 85, 4837, 2004.

[6] B. He, Q. Lou, J. Zhou, J. Dong, Y. Wei, D. Xue, Y. Qi, Z. Su, L. Li, and F. Zhang. Opt. Exp., 14, 2721, 2006.

[7] T. H. Loftus, A. M. Thomas, M. Norsen, J. Minelly, P. Jones, E. Honea, S. A. Shakir, W. Culver, and Y. Stracher. Proc. of Solid State Diode Laser Technology Review, June 2006.

[8] V. A. Kozlov, J. Hernandez-Cordero, and T. F. Morse. Opt. Lett., 24, 1814, 1999.

[9] A. Shirakawa, T. Saitou, T. Sekiguchi, and K. -I. Ueda. Opt. Exp., 10, 1167, 2002. 
[10] L. Shimshi, A. A. Ishaayaa, N. Davidson, A. A. Friesem. Opt. Comm., 275 389, (2007).

[11] V. Eckhouse, A. A. Ishaaya, L. Shimshi, N. Davidson, and A. A. Friesem. Opt. Lett., 31, 350, 2006.

[12] M. Fridman, V. Eckhouse, N. Davidson, and A. A. Friesem. Opt. Lett., 1, 790, 2007.

[13] V. Eckhouse, M. Fridman, N. Davidson, and A. A. Friesem. Phys. Rev. Lett., 100, 024102, (2008).

[14] J. E. Rothenberg. Proc. of SPIE August 12-14 2008, 6873, 15, (2008). 